\title{
Evaluation of Proinflammatory Cytokines and Adverse Events in Healthy Volunteers upon Inhalation of Antituberculosis Drugs
}

\author{
Teerapol Srichana, ${ }^{*, a}$ Chaveewan Ratanajamit, ${ }^{b}$ Siwasak Juthong, ${ }^{c}$ Tan Suwandecha,,${ }^{a}$, \\ Nisa Laohapojanart, ${ }^{d}$ Petchawan Pungrassami, ${ }^{e}$ and Alwar Ramanujam Padmavathi ${ }^{a}$ \\ ${ }^{a}$ Nanotec-PSU Center of Excellence on Drug Delivery System and Department of Pharmaceutical Technology, \\ Faculty of Pharmaceutical Sciences, Prince of Songkla University; Hat Yai, Songkhla 90112, Thailand: ${ }^{b}$ Department \\ of Clinical Pharmacy, Faculty of Pharmaceutical Sciences, Prince of Songkla University; Hat Yai, Songkhla 90112, \\ Thailand: ${ }^{c}$ Department of Internal Medicine, Faculty of Medicine, Prince of Songkla University; Hat Yai, Songkhla \\ 90112, Thailand: ${ }^{d}$ Department of Pharmacy, Chulalongkorn Hospital; Bangkok 10330, Thailand: and ${ }^{e}$ Bureau of \\ Tuberculosis, Department of Disease Control, Ministry of Public Health; Bangkok 10120, Thailand. \\ Received April 22, 2016; accepted August 12, 2016
}

\begin{abstract}
Inhalation therapy is a promising drug delivery approach for tuberculosis treatment. However, there is always concern about the safety of the dosage form by inhalation as it may induce inflammation. Developing a new dosage form for inhalation must include tests for its safety especially for the tumor necrosis factor (TNF)- $\alpha$ and interleukine (IL)-1 $\beta$. The safety of four anti-tuberculosis (anti-TB) drugs administered via inhalation was assessed in healthy volunteers. Four anti-TB drugs; isoniazid, rifampicin, pyrazinamide and levofloxacin were prepared as dry powder and evaluated for uniformity of delivered dose and in vitro drug deposition. These four anti-TB dry powder formulations for inhalation met the criteria of uniformity of delivered dose and exhibited suitable size for lung delivery. Forty healthy volunteers were recruited and each was sequentially challenged with isoniazid, rifampicin, pyrazinamide and levofloxacin in different orders. Safety was monitored by measuring the pro-inflammatory cytokines in their sputum, lung function test, blood chemistry and adverse events. This study proves that all four anti-TB dry powders did not provoke inflammatory cytokines and are safe to healthy volunteers.
\end{abstract}

Key words antituberculosis; clinical trial; pulmonary drug delivery; dry powder inhalation; toxicity; cytokine

Tuberculosis (TB) is a deadly disease which killed nearly 1.5 million people during 2014. ${ }^{1)}$ TB remains to be one of the world's biggest threats, for which a long-term treatment for cure is the most desirable objective and it often comes with few side effects. ${ }^{2)}$ Long-term treatment of anti-TB drugs at higher concentration leads to these side effects. Delivery of drugs directly to the target site may help to minimize the dosage and subsequent side effects. Pulmonary drug delivery has been widely accepted as an alternate route of treatment. Delivery of drug by inhalation also has considerable challenges in getting the drug to its target site because of the complexity of the lungs. ${ }^{3-5)}$ Furthermore, the lungs may change dramatically when afflicted by disease. Drug delivery to the deep lung may be impeded by changes such as mucus hyper-secretion or by the thickening and narrowing of the airways. ${ }^{6}$

Proper research and interpretation are important to achieve better delivery of anti-TB drugs to human lungs. However, first-line anti-TB drugs used for inhalation have been evaluated for their safety only in cell lines and with some in vivo animal models. $^{7-10)}$ Clinical trials are crucial to completely evaluate the effects of anti-TB drugs on the lungs and to establish a successful therapeutic route to achieve maximum recovery from TB. Clinical trial phase I carried out with capreomycin to treat highly drug resistant $\mathrm{TB}$, indicated that inhalation of capreomycin was safe and advisable for TB therapy. ${ }^{11)}$ Accurate data interpretation is important to assess the effects of

${ }^{\dagger}$ Present address: Department of Pharmacology, Faculty of Science, Prince of Songkla University; Hat Yai, Songkhla 90112, Thailand. drugs in clinical trials. It plays an important role to determine the outcome of treatment. In order to interpret safety data, it is necessary to reconcile test sensitivity, variations in different biological backgrounds with normal responses to the inhaled materials and drug-specific adverse effects. Identification of specific adverse end-points can be determined by better controlled study to discern true adverse effects from normal physiological lung responses. This can only be achieved by performing clinical trial phase I with healthy volunteers to record the normal physiological responses.

The lung responds acutely to irritant materials by hypersecretion of mucus, release of chemokines, recruitment of inflammatory cells and cough reflexes. But most of these responses are considered as non-specific defenses. ${ }^{12,13)}$ Sputum cytokines are specific indicators of acute inflammation of the airways. ${ }^{14)}$ Lipopolysaccharide-induced lung toxicity studies have revealed the significant increase of interleukin (IL)- $1 \beta$, IL-6, and tumor necrosis factor (TNF)- $\alpha$ level in sputum. ${ }^{15,16)}$ Beside these, the correlation between the presence of cytokines in exhaled breath condensate and airways inflammation was previously well established. ${ }^{17,18)}$ The cytokines were exploited as safety criteria in this study to evaluate the effect of anti-TB formulations on healthy volunteers.

Isoniazid, rifampicin, and pyrazinamide are the core drugs used in standard short course regimens for emerging $\mathrm{TB}$ patients in Thailand. In addition to these, levofloxacin is a drug used in standard regimens for patients with multi-drug resistant (MDR)-TB infections and WHO also initiated the evaluation of levofloxacin with other new anti-TB drugs to 
Table 1. Dose Calculation of Antituberculosis Dry Powder Formulations (R, Rifampicin; H, Isoniazid; Z, Pyrazinamide; Lfx, Levofloxacin)

\begin{tabular}{|c|c|c|c|c|c|c|}
\hline & $\mathrm{R}$ & $\mathrm{H}$ & $\mathrm{Z}$ & Lfx & Reference(s) & Dosage form \\
\hline Dose (mg/kg) & $4-6$ & $8-12$ & $20-30$ & $9-16$ & 28 & Oral \\
\hline $\mathrm{MIC}(\mu \mathrm{g} / \mathrm{mL})$ & 0.6 & 0.4 & 50 & 1 & & \\
\hline$C_{\max }(\mu \mathrm{g} / \mathrm{mL})$ & $5-10$ & $4-5$ & $30-50$ & $2-3$ & $29-32$ & \\
\hline Dose $(\mu \mathrm{g})^{a)}$ & $75-150$ & $60-75$ & $450-750$ & $30-45$ & & Inhaled \\
\hline Cal. dose $\left.(\mathrm{mg})^{b}\right)$ & $0.75-1.5$ & $0.6-0.75$ & $4.5-7.5$ & $0.3-0.45$ & & Formulation \\
\hline Adj. dose $(m g)^{c}$ & 2 & 5 & 16 & 2 & & \\
\hline
\end{tabular}

$C_{\max }$-Maximum serum concentration; Dose—Dose theoretically required; Cal. dose—Calculated dose; Adj. dose—Adjusted dose. a) Based on MIC $\times 10$ times $\times 15 \mathrm{~mL}$ lung fluid or $C_{\max } \times 15 \mathrm{~mL}$ lung fluid. $b$ ) Calculated dose on $10 \%$ fine particle fraction (Delivery efficiency). $c$ ) Escalated dose for free water soluble Isoniazid and Pyrazinamide.

treat MDR-TB. According to Thai Clinical Practice Guidelines for the Treatment of Tuberculosis in adults, the oral dosage of isoniazid, rifampicin and pyrazinamide are 300, 600 and $1500 \mathrm{mg} / \mathrm{d}$ respectively. Due to the administration of high dose, these drugs are prone to elicit severe adverse effects that include hepatotoxicity, neurotoxicity and nephrotoxicity. An alternative dosage form such as using a dry powder inhaler can reduce the dose and can potentially be applied to treat patients with TB lesions in the lungs. This would be a preferred option to improve the treatment efficacy without compromising patients' tolerance and safety.

This study was intended to evaluate the safety of 4 anti-TB drugs (isoniazid, rifampicin, pyrazinamide, levofloxacin) in dry powder formulations designed to use as multiple low dose respiratory agents. The dry powder formulations used in this study were formulated using liposomal-based drug delivery systems to target the alveolar macrophages. ${ }^{7,8,10,19)}$

\section{MATERIALS AND METHODS}

Study Hypothesis Anti-TB dry powder inhalers cause no or minimal adverse reactions to the respiratory tract and are therefore safe to use. As the inhalation dose was very low, it was not expected to cause hepatotoxicity and nephrotoxicity and it will not elicit adverse immune responses. ${ }^{20)}$

Anti-TB Dry Powder Formulations The amount of drugs adapted from available literature and the dosage regimen calculation are shown in Table $1 .^{8,10,21-23)}$ The adjusted doses of the four drugs are 2-7 times of the calculated doses according to different drug potency $(2-16 \mathrm{mg})$. These were done to compromise the feasible fixed dose combination of anti-TB for inhalation which should not be too high or too low. ${ }^{5}$ All chemicals used in this study were of pharmaceutical grade. Isoniazid was obtained from Honor Pharm (Shanghai) Co., Ltd., China; batch no. P102808. Rifampicin was obtained from Linaria Chemical (Thailand) Ltd., Thailand; batch no. RMP/ XP-002/12 (Crystalline). Pyrazinamide was obtained from Lupin Ltd., India; batch no. 120942187. Levofloxacin was obtained from Macleods Pharmaceuticals Ltd., India; batch no. E/6800/13001. Four anti-TB powder formulations were manufactured separately according to the Thai current Good Manufacturing Practice by mixing respective drugs (isoniazid $5 \mathrm{mg}$, rifampicin $2 \mathrm{mg}$, pyrazinamide $16 \mathrm{mg}$ or levofloxacin $2 \mathrm{mg}$ ) with mannitol, cholesterol $(\mathrm{CH})$ and soybean phosphatidylcholine (SPC) (Table 2). Briefly SPC and $\mathrm{CH}$ were dissolved in hydroalcohol containing ethanol and water $(2: 1)$ until a clear solution was formed. Then mannitol spheres of $2 \mu \mathrm{m}$ were suspended in the hydroalcoholic solution followed by the addition
Table 2. Contents of a Capsule Containing Antituberculosis Dry Powder Formulation

\begin{tabular}{lrrrr}
\hline \hline \multirow{2}{*}{ Ingredient (mg) } & \multicolumn{4}{c}{ Formulation } \\
& $\mathrm{A}(\mathrm{H})$ & $\mathrm{B}(\mathrm{Lfx})$ & $\mathrm{C}(\mathrm{Z})$ & $\mathrm{D}(\mathrm{R})$ \\
\hline Antibiotics & 5 & 2 & 16 & 2 \\
Mannitol & 20 & 23 & 9 & 23 \\
Phosphatidylcholine & 2 & 2 & 2 & 2 \\
Cholesterol & 1 & 1 & 1 & 1 \\
\hline
\end{tabular}

R, Rifampicin; H, Isoniazid; Z, Pyrazinamide; Lfx, Levofloxacin.

of anti-TB drugs to their respective mixture. These suspensions were spray dried at an inlet temperature of $100^{\circ} \mathrm{C}$ with a spray rate of $3 \mathrm{~mL} / \mathrm{min}$ until the dried powder formulations were obtained. The dried formulations were precisely weighed for $28.7 \mathrm{mg}$ and filled into individual No. 3 capsules.

Analysis of the Anti-TB Powder Formulations Contents of anti-TB formulations (to determine the concentration of isoniazid, rifampicin, pyrazinamide and levofloxacin) were analyzed using HPLC (SpectraSYSTEM ${ }^{\mathrm{TM}}$, Thermo Fisher Scientific, Inc., U.S.A.) equipped with a UV detector. A BDS Hypersil C18 column $(150 \times 4.6 \mathrm{~mm}$ i.d., $5 \mu \mathrm{m})$ (Thermo Scientific, U.K.) was used with the mobile phase $25 \mathrm{~mm}$ phosphate buffer ( $\mathrm{pH}$ 3.2) and acetonitrile in the ratio of $65: 35(\mathrm{v} / \mathrm{v})$ with a flow rate of $1.5 \mathrm{~mL} / \mathrm{min}$ at ambient temperature. The UV detector was operated at $254 \mathrm{~nm}$.

Uniformity of Delivered Dose The uniformity of delivered dose from the dry powder inhaler (DPI) was assessed using the apparatus specified in the British Pharmacopoeia. ${ }^{24)}$ The DPI devices used in this study were designed in house (Dynamic Promotion, Thailand). The drug content of the DPI was delivered into the Andersen Cascade Impactor (ACI) and was quantitatively collected and analyzed for the amount of each anti-TB agent.

The In-House Designed DPI Device The in-house designed DPI device was made of polypropylene plastic. The geometry of the device was a cylindrical inhaler body with semi-elliptical frustum mouthpieces (Fig. 1). There was a capsule port and two circular bleed holes at the end of the cylindrical body. The grid was placed between the inhaler body and mouthpiece to prevent capsule debris leaving the inhaler. The device was classified as a low resistance device with a pressure drop of 0.1 (mbar) $)^{1 / 2} /$ litre per minute (LPM). ${ }^{25,26)}$

Assessment of Distribution of Aerodynamic Particle Size To measure the aerodynamic particle size, an eight-stage ACI equipped with a pre-separator (Atlanta, GA, U.S.A.) was employed. The capsule containing the anti-TB drug was in- 

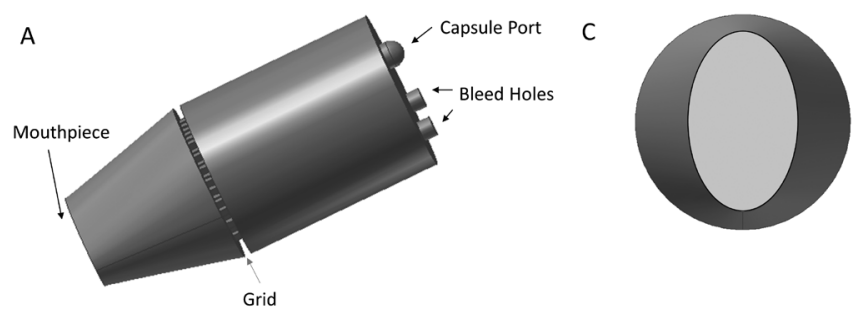

B

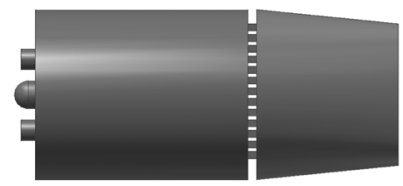

D

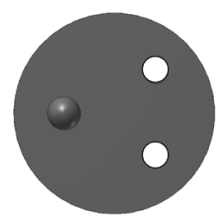

Fig. 1. The Internal Geometry of the In-House Designed Dry Powder Inhaler Device

Isometric view (A), side view (B), top view (C), bottom view (D).

serted into the inhaler. The inhaler was twisted to separate the capsule before it was introduced into the impactor. Air was drawn through the apparatus and the flow rate was adjusted to $60 \mathrm{~L} / \mathrm{min}$. Each fraction of drug deposition was adjusted to a specified volume and analyzed by HPLC. The mass median aerodynamic diameter (MMAD) and the fine particle fraction (FPF) were calculated according to the method described by Srichana et al. $^{26)}$

Study Design This was a double-blinded four-treatment, four-period crossover design, with a washout period of 1 week between treatments. The study took place at the clinical laboratory, Faculty of Pharmaceutical Sciences, Prince of Songkla University, from February-March 2013. Forty (20 male and 20 female) healthy subjects were selected for the study. The sample size was chosen based on the guidance of clinical trial phase I. ${ }^{27)}$

Participants were healthy adults who met the following eligibility criteria: aged 18-30 years; body mass index $18-27 \mathrm{~kg}$ / $\mathrm{m}^{2}$; assessed as being healthy by medical history, physical examination, vital signs and normal clinical laboratory results (blood urea nitrogen [BUN], serum creatinine [Cr], aspartate transaminase $[\mathrm{AST}](<2$ fold of the upper normal value), alanine transaminase $[\mathrm{ALT}](<2$ fold of the upper normal value), total bilirubin, alkaline phosphatase [ALP], blood potassium, sodium, calcium, chloride, fasting blood sugar, hematology examinations including complete blood counts, chest radiography and negative HBsAg.

The exclusion criteria were: history of allergies to any antiTB drugs or other components; high blood pressure; underlying diseases such as asthma, chronic obstructive pulmonary disease, chronic kidney disease, diabetes mellitus, liver diseases, immunodeficiency, hepatitis, cough and cold; smokers $>10$ cigarettes/d or smokers $<10$ cigarettes/d who could not quit for at least $7 \mathrm{~d}$ before the study and throughout the study (including the washout periods); regular alcohol consumption (more than 1 time/week) or alcohol consumption within $7 \mathrm{~d}$ prior to the study. All subjects had a chest X-ray (CXR) during the screening visit. In the case of reproductive age women, negative urine pregnancy test at screening, non-lactating women, and use of effective contraceptives for at least 4 weeks before the screening examination until the end of the study were required.
A balanced Latin square block randomization was used in this study to achieve a balancing of order (sequence) to avoid the period effects. Each formulation was assigned the code A (isoniazid), B (levofloxacin), C (pyrazinamide), and D (rifampicin), and the treatment sequences were generated as $\mathrm{ABCD}$ (sequence 1), BCDA (sequence 2), $\mathrm{CDAB}$ (sequence 3) and DABC (sequence 4). On the first day of the drug dosing in period 1, participants were stratified by gender and randomly assigned into one of the four treatment sequences, as indicated in the pre-printed randomization scheme with the block size of 4 , and the allocation ratio of $1: 1: 1: 1$. Subjects in sequence 1 received treatment $\mathrm{A}$ during the first study period and then crossed over to receive treatment B, C, and D at the second, third and fourth periods respectively (after the 7-d washout period).

Intervention All the subjects were trained to self-administer the DPI and peak flow meter before receiving the study drugs. Four anti-TB formulations (isoniazid $5 \mathrm{mg}$, rifampicin $2 \mathrm{mg}$, pyrazinamide $16 \mathrm{mg}$, and levofloxacin $2 \mathrm{mg}$ ) were administered to each patient according to the assigned treatment sequence. In each period, each subject received the assigned formulation for 7 consecutive days, at the dosing interval of $24 \pm 1 \mathrm{~h}$. Two pharmacists were responsible for the administration of the drug. No other medications were allowed during the entire study period.

Safety Evaluation of the Antituberculosis Dry Powder Inhalers The study protocol was approved by the Ethics Committee (EC), Ministry of Public Health, Thailand (RES. No. 18/2554; approved on 25 January, 2012). The protocol was also submitted to the United States Food and Drug Administration (U.S. FDA) clinical trials (NCT01785927). The study was conducted in accordance with the Declaration of Helsinki and principles of Good Clinical Practice. Subjects provided written informed consent before participating in this study procedure. For safety evaluation purposes, induced sputum samples by normal saline solution were taken from subjects before the commencement of the inhalation to obtain baseline for cytokines. After inhalation of medication, the subjects were asked to rinse their mouth with $50 \mathrm{~mL}$ of water and expectorate deeply to collect $1 \mathrm{~mL}$ of sputum sample into wide covered sterile $50 \mathrm{~mL}$ plastic cups. The collected samples were immediately transferred to laboratory for cytokines testing and analyzed within $1 \mathrm{~h}$. The TNF- $\alpha$ and IL- $1 \beta$ were detected using enzyme-linked immunosorbent assay (ELISA) kits (R\&D Systems, MN, U.S.A.) with a detection limit of $5 \mathrm{pg} / \mathrm{mL}$.

Safety Outcomes Inflammatory cytokines (TNF- $\alpha$, IL-1 $\beta$ ) were measured at day 1 (prior to drug administration as baseline), days 4 and 7 of each study period and considered as the primary outcomes. Secondary outcomes were the liver function test performed after the last dosing (day 7) of each study period. Other secondary outcomes were adverse events (AEs) reported during the trial, and expiratory peak flow values during each clinical visit. Subjects were monitored for AEs, and interviewed for any illnesses prior to each dosing of drug. AEs were recorded in the source document and evaluated for their severity, and relationship with the medications used in this study by a clinical investigator. All serious AEs, if occurred, were reported to the EC within 1 week. A post-study safety evaluation for all subjects was conducted by physical examinations, vital sign measurements, blood chemistry (liver 
function, renal function tests), complete blood counts, and a CXR at the end of the study.

Statistical Analysis Data were computerized and analyzed with SPSS software Version 17.0 (IBM, U.S.A.). The baseline parameters were summarized. Continuous variables were presented as the mean \pm standard deviation (S.D.), while categorical variables were presented as absolute values and relative frequencies (\%). The analyses were based on the intention-to-treat (ITT) population. The primary outcomes at the end of each study period were compared with the baseline values using the paired $t$-test. The Wilcoxon signed-rank tests were used in case of any non-normally distributed data. Box-and-whisker plot was constructed to show distribution of data. The upper and the lower ends of the whiskers are; third quartile plus 1.5 interquartile range and first quartile minus 1.5 interquartile range, respectively. Values beyond either the upper end or the lower end of whiskers are considered outliers. Statistical significance was set as $p<0.05$.

\section{RESULTS}

Uniformity of the Delivered Dose from the Antituberculosis Dry Powder Inhalers The percentage labeled amount of isoniazid, rifampicin, pyrazinamide and levofloxacin were $114.0 \pm 0.7,101.4 \pm 2.2,97.8 \pm 4.8$ and $117.2 \pm 0.7$, respectively (Table 3). The corresponding percentage delivered doses were 102.2 $\pm 1.9,87.9 \pm 1.2,89.2 \pm 0.8$ and 97.4 \pm 1.4 (Table 3). All formulations satisfied the criteria for the uniformity of the delivered dose as suggested by British Pharmacopoeia. ${ }^{24}$

In Vitro Deposition of Drugs by ACI The MMADs for isoniazid, rifampicin, pyrazinamide and levofloxacin were $3.0 \pm 0.4,2.2 \pm 0.1,4.3 \pm 0.2$ and $4.1 \pm 0.1 \mu \mathrm{m}$, respectively. The percentage of fine particle fractions (FPFs) for isoniazid, rifampicin, pyrazinamide and levofloxacin were 35.0 \pm 4.0 ,
$37.4 \pm 2.0,29.0 \pm 4.1$ and 28.1 \pm 4.6 , respectively (Table 3). All formulations exhibited FPFs and mass median aerodynamic diameters (MMAD) that met the standards specified in the British Pharmacopoeia ${ }^{24)}(>25 \%)$ and there were no significant differences in the values of FPF and MMAD between isoniazid and rifampicin, and between pyrazinamide and levofloxacin $(p>0.05)$.

Safety Evaluation of Antituberculosis Dry Powder Inhalers in Vivo Of the 45 subjects screened, 40 healthy subjects were included in the study. A total of 5 subjects were excluded from the study for various reasons (Fig. 2). The enrollment details of the study subjects were summarized in Fig. 2. Forty subjects were randomized to the treatment sequences, but 1 subject was lost to follow-up during randomization. The remaining 39 subjects completed the four-period, four-treatment crossover study. The mean age and weight of the subjects were 22.5 years and $58.4 \mathrm{~kg}$, respectively. There was no statistically significant difference in the baseline characteristics between the male and female groups $(p>0.05)$. Blood chemistry levels at baseline revealed that all participants had normal kidney and liver functions (Table 4).

However, baseline cytokine levels among the subjects were within the normal range, but distribution of the data was not normal. Therefore, the Wilcoxon signed rank test was used to

Table 3. Characteristics of the Antituberculosis Dry Powder Formulations Used for Inhalation (Mean \pm S.D., $n=6$ )

\begin{tabular}{lccc}
\hline \hline Active ingredients & \% Delivered dose & MMAD $(\mu \mathrm{m})$ & $\%$ FPF \\
\hline Isoniazid & $102.2 \pm 1.9$ & $3.2 \pm 0.4$ & $35.0 \pm 4.0$ \\
Rifampicin & $101.4 \pm 2.2$ & $3.0 \pm 0.1$ & $37.4 \pm 2.0$ \\
Pyrazinamide & $97.8 \pm 4.8$ & $4.3 \pm 0.2$ & $29.0 \pm 4.1$ \\
Levofloxacin & $97.4 \pm 1.4$ & $4.1 \pm 0.1$ & $28.1 \pm 4.6$ \\
\hline
\end{tabular}

MMAD, Mass median aerodynamic diameter; FPF, Fine particle fraction.

Table 4. Blood Chemistry of the Volunteers Prior to the Clinical Study $(n=39)$

\begin{tabular}{|c|c|c|c|c|c|c|}
\hline Variables & Mean \pm S.D. & Median & Min & Max & Normal value & Units \\
\hline Hemoglobin & $13.7 \pm 1.9$ & 13.7 & 9.2 & 16.8 & $13.0-18.0$ & $\mathrm{~g} / \mathrm{dL}$ \\
\hline Hematocrit & $40.9 \pm 5.0$ & 41.0 & 29.9 & 50.3 & $40.0-54.0$ & $\%$ \\
\hline Red blood cells & $4.9 \pm 0.5$ & 5.1 & 4.1 & 6.1 & $4.5-6.3$ & $10^{3} / \mathrm{dL}$ \\
\hline $\mathrm{MCV}$ & $82.0 \pm 7.0$ & 83.9 & 59.2 & 90.3 & $83.0-97.0$ & $\mathrm{fL}$ \\
\hline $\mathrm{MCH}$ & $27.4 \pm 3.1$ & 28.4 & 18.2 & 31.5 & $27.0-33.0$ & pg \\
\hline $\mathrm{MCHC}$ & $33.4 \pm 1.3$ & 33.5 & 30.7 & 36.7 & $31.0-35.0$ & $\mathrm{~g} / \mathrm{dL}$ \\
\hline RDW & $13.3 \pm 2.0$ & 12.9 & 10.3 & 19.6 & $11.0-16.0$ & $\%$ \\
\hline White blood cells & $7.2 \pm 2.5$ & 6.6 & 4.4 & 19.3 & $4.5-10.0$ & $10^{3} / \mathrm{dL}$ \\
\hline PMN & $57.0 \pm 10.7$ & 56.5 & 38.0 & 94 & $40-70$ & $\%$ \\
\hline Eosinophils & $3.3 \pm 2.3$ & 2.7 & 0.1 & 8.8 & $1.0-6.0$ & $\%$ \\
\hline Basophils & $0.6 \pm 0.6$ & 0.3 & 0.1 & 3.0 & $0.0-1.0$ & $\%$ \\
\hline Lymphocytes & $33.4 \pm 9.6$ & 33.3 & 2.8 & 50.0 & $20.0-50.0$ & $\%$ \\
\hline Monocytes & $6.1 \pm 2.1$ & 5.5 & 3.0 & 12.0 & $2.0-10.0$ & $\%$ \\
\hline Platelets & $262.8 \pm 44.4$ & 258.0 & 190.0 & 382.0 & $150-450$ & $10^{3} / \mathrm{dL}$ \\
\hline BUN & $11.8 \pm 2.3$ & 11.5 & 6.5 & 16.8 & $5.0-23.0$ & $\%$ \\
\hline Uric acid & $5.5 \pm 1.3$ & 5.6 & 2.6 & 8.6 & $3.4-7.0$ & $\mathrm{mg} \%$ \\
\hline Initial serum creatinine & $0.8 \pm 0.2$ & 0.8 & 0.5 & 1.1 & $0.5-1.2$ & $\mathrm{mg} \%$ \\
\hline Post serum creatinine & $0.9 \pm 0.2$ & 0.9 & 0.6 & 1.2 & $0.5-1.2$ & $\mathrm{mg} \%$ \\
\hline AST & $25 \pm 25$ & 19.0 & 13.0 & 36.0 & $10-35$ & $\mathrm{u} / \mathrm{L}$ \\
\hline ALT & $21 \pm 15$ & 18.0 & 8.0 & 41.0 & $9-40$ & $\mathrm{u} / \mathrm{L}$ \\
\hline ALP & $73 \pm 19$ & 70.0 & 36.0 & 124.0 & $30-120$ & $\mathrm{u} / \mathrm{L}$ \\
\hline
\end{tabular}

MCV, Mean corpuscular volume; MCH, Mean corpuscular hemoglobin; MCHC, Mean corpuscular hemoglobin concentration; RDW, Red cell distribution width; PMN, Polymorphonuclear leukocytes; BUN, Blood urea nitrogen; AST, Aspartate aminotransferase; ALT, Alanine aminotransferase; ALP, Alkaline phosphatase. 
compare the inflammatory cytokine levels prior and later to drug administration. The IL-1 $\beta$ level in the sputum of healthy volunteers after inhalation of isoniazid, rifampicin, pyrazinamide and levofloxacin at different study periods (Fig. 3A) revealed the increase in IL- $1 \beta$ level from days 1 to 4 and from days 1 to 7 with no significance $(p>0.05)$. Even though the cumulative cytokine levels (Fig. 3A) were less than $1000 \mathrm{pg} /$ $\mathrm{mL}$, few volunteers had a common cold during the study which resulted in increased IL- $\beta(>1000 \mathrm{pg} / \mathrm{mL})$. TNF- $\alpha$ levels (Fig. 3B) were also found to be increased from days 1 to 4 during each treatment but no significance was observed $(p>0.05)$.

Majority of the volunteers (85-90\%) were devoid of any adverse events after each treatment. Only a small number $(10-15 \%)$ of subjects reported minor adverse effects. Safety evaluation of the liver function by AST, ALT, ALP, Total bilirubin (TB) and Direct bilirubin (DB) revealed that all the liver enzymes were within normal limits before and after each period (Table 5). The creatinine level at the end of the study was also within the normal limit (Table 4). It indicated that the anti-TB formulations did not affect the kidney functions.
All subjects had a normal peak expiratory flow rate before and after receiving all the anti-TB formulations (Table 6). There was no complaint or clinical observation that the subjects had nausea/vomiting, anorexia, bilirubinemia and jaundice after administration of the anti-TB dry powders. No serious adverse events were noted (Table 7). A fever and fatigue was reported from one subject who had a common cold during the study. The symptom was relieved after $4-7 \mathrm{~d}$ when the subject recovered from the common cold.

\section{DISCUSSION}

Local delivery of a drug to the lung for the treatment of pulmonary TB may enhance the therapeutic index by targeting the macrophages in which the TB bacilli reside. ${ }^{21)}$ However, clinical studies are necessary to ensure the safety of inhaled anti-TB dry powders alongside to their better clinical efficacy. Safety concerns of local delivery to the lung include local lung effects and systemic side effects such as activation of immune responses. Local irritant effects resulting from the inhalation anti-TB drugs can be clinically manifested by

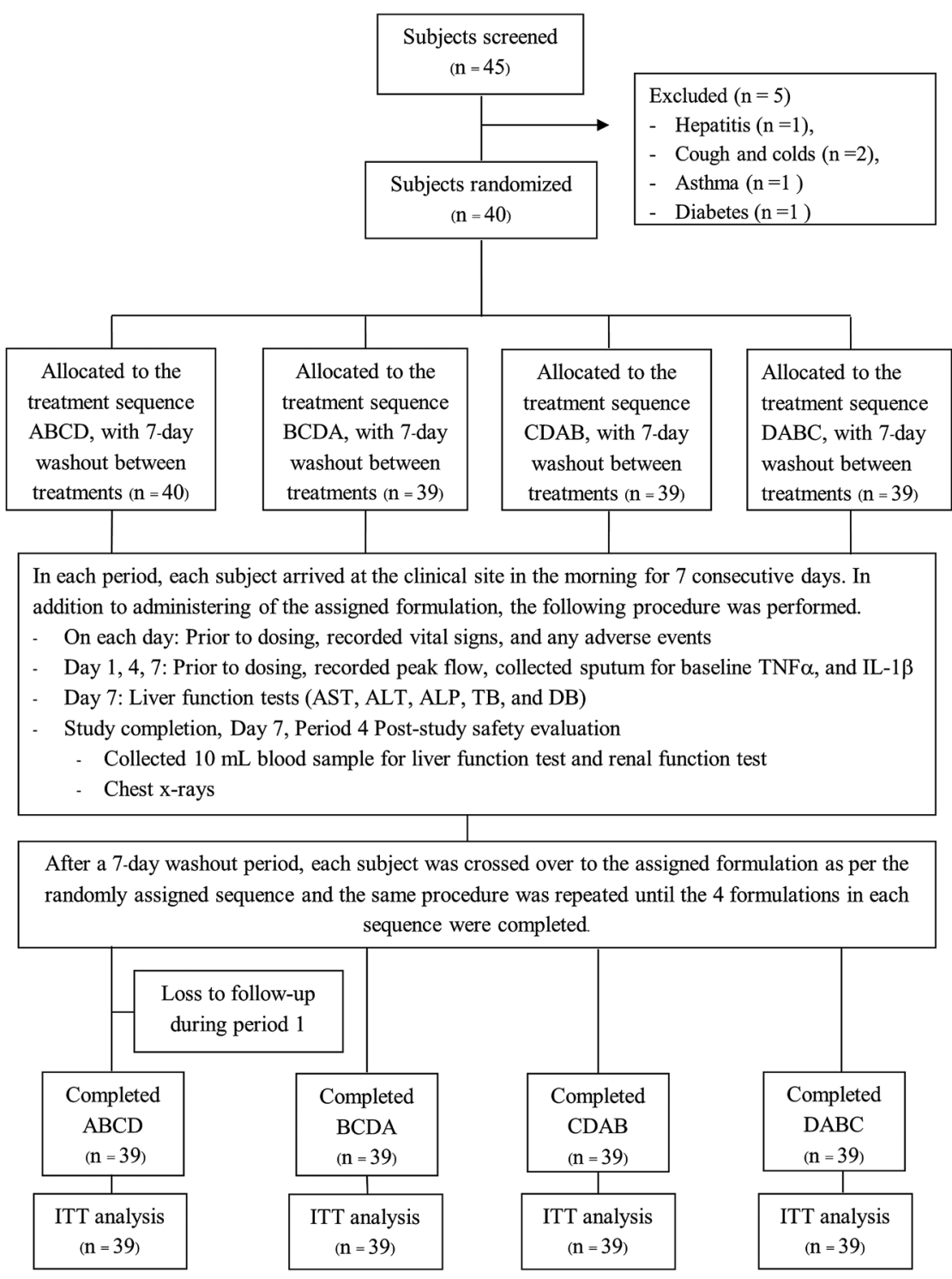

Fig. 2. Flow Chart Depicting the Study Design 
A
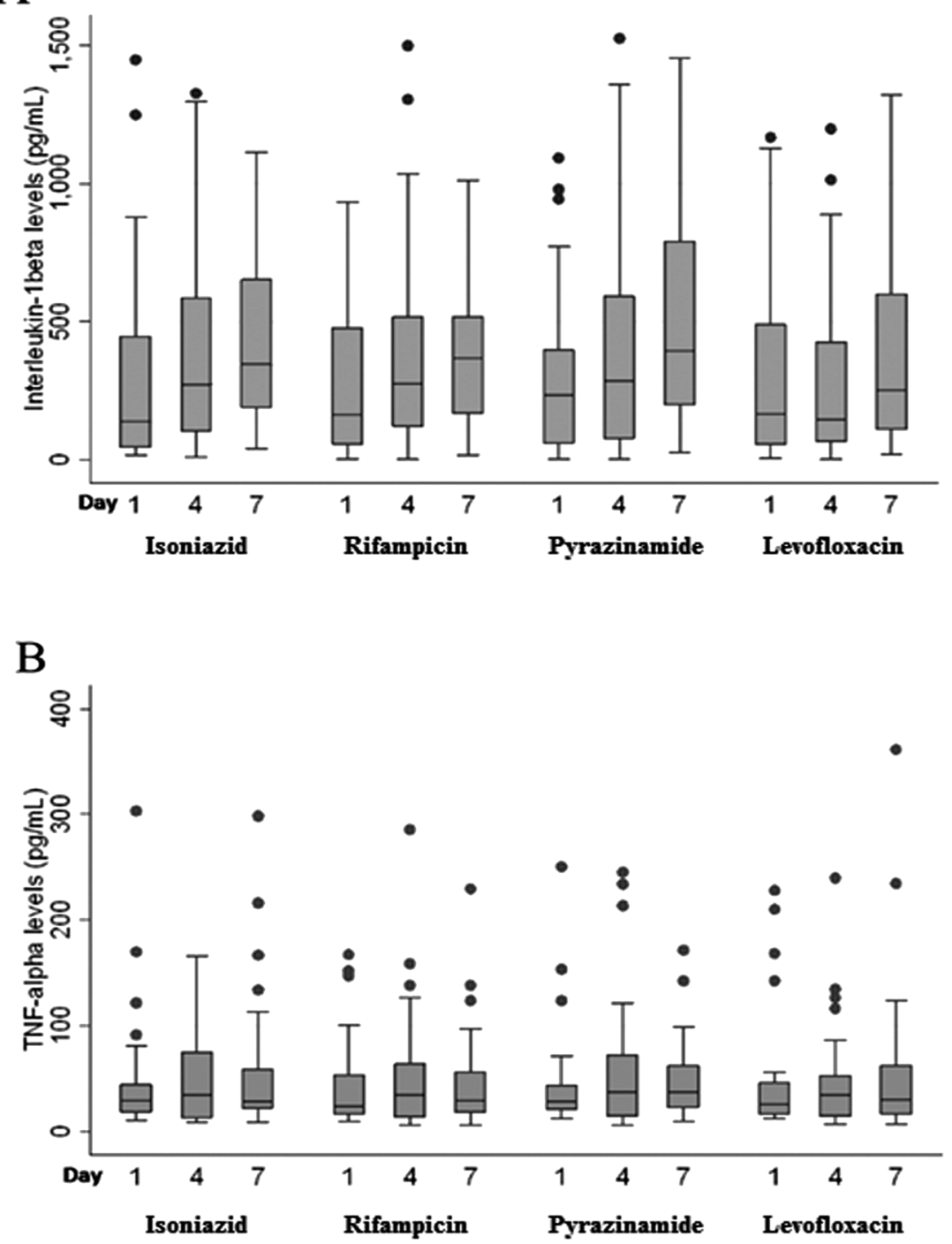

Fig. 3. The Box-and-Whisker Plot Showing the Distribution of IL-1 $\beta$ Levels (A) and TNF- $\alpha$ Levels (B) at Days 1,4 and 7 in the Sputum of Subjects $(N=39)$ after Administering Isoniazid, Rifampicin, Pyrazinamide and Levofloxacin Dry Powders to the Lung

The upper and the lower ends of the whiskers are third quartile +1.5 interquartile range and first quartile -1.5 interquartile range, respectively. Values beyond either the upper end or the lower end of whiskers are considered outliers.

a cough and bronchospasm. There is no clinically significant cough and bronchospasm were reported in this study following inhalation of the anti-TB dry powders. Systemic allergic or hypersensitivity reactions were also not observed in the subjects. TNF- $\alpha$ in all subjects remained unchanged $(p>0.05)$ although the cytokines slightly increased. The level of IL- $1 \beta$ increased significantly to $1000 \mathrm{pg} / \mathrm{mL}$ but afterwards it changed little. However, the median ranges were within the safety window. Longer treatment periods further increased the level of cytokines but not significantly. This study has shown that the cytokines at these levels did not cascade into severe inflammation. Adverse events such as fever were observed in one subject but it was speculated due to a secondary viral infection. There were no clinically significant respiratory adverse events reported. For example, shortness of breath, more difficulty to breath except that one subject receiving pyrazinamide had a very high TNF- $\alpha(1358.4 \mathrm{pg} / \mathrm{mL})$ prior to and after administration $(2109.8 \mathrm{pg} / \mathrm{mL})$. This particular subject reported to the clinical investigator that he had a very bad cold followed by nausea, cough, muscle cramp and fever.
These data points were excluded as they were the resultant of cold and associated symptoms and not because of the drug. However, this TNF- $\alpha$ returned to normal at day $7(99.1 \mathrm{pg} / \mathrm{mL})$ when the cold was relieved. This different result was expected to be the result of a viral infection that had caused a dramatic increase in the production of the cytokines (TNF- $\alpha$ and IL-1 $\beta$ ). In addition, the common cold symptom was not persistent in the subjects; it was relieved after $4-7 \mathrm{~d}$. The outliers of each period varied from 1-4 out of 39 volunteers. Even then, they are still inside the safety window and the values are not statistically significant.

There was neither any toxicity related to the inhaled antiTB drugs observed in this study nor were there any effects on the renal functions, liver functions and other systemic parameters that all remained within the normal ranges. For safety consideration of the kidney, a serum creatinine test was carried out prior to enrolling into the study and after the study period which found to be normal in all the subjects (Table 4). The assertion of safety was based on the dosage and outcome measures in this study. There was a minor evidence of adverse 
Table 5. Secondary Safety Outcome Data of Volunteers during Screening Examination and after Administration of Isoniazid, Rifampicin, Pyrazinamide and Levofloxacin by Inhalation

\begin{tabular}{|c|c|c|c|c|c|}
\hline \multirow{2}{*}{ Variables } & \multicolumn{5}{|c|}{ Mean \pm S.D., Median, $[\min -\max ], n=39$} \\
\hline & Baseline & Isoniazid & Rifampicin & Pyrazinamide & Levofloxacin \\
\hline AST (U/L) & $\begin{array}{c}24.7 \pm 24.9,19.0 \\
{[13.0-32.0]}\end{array}$ & $\begin{array}{c}21.6 \pm 7.6,18.0 \\
{[14.0-49.0]}\end{array}$ & $\begin{array}{c}19.7 \pm 4.0,19.0 \\
{[13.0-30.0]}\end{array}$ & $\begin{array}{c}21.3 \pm 5.5,19.0 \\
{[12.0-66.0]}\end{array}$ & $\begin{array}{c}19.8 \pm 5.5,19.0 \\
{[13.0-43.0]}\end{array}$ \\
\hline ALT (U/L) & $\begin{array}{c}20.8 \pm 14.7,18.0 \\
{[8.0-46.0]}\end{array}$ & $\begin{array}{c}21.2 \pm 16.9,15.0 \\
{[8.0-49.0]}\end{array}$ & $\begin{array}{c}19.8 \pm 12.4,16.0 \\
{[7.0-36.0]}\end{array}$ & $\begin{array}{c}21.2 \pm 15.5,16.0 \\
{[8.0-45.0]}\end{array}$ & $\begin{array}{c}21.7 \pm 23.1,16.0 \\
{[8.0-49.0]}\end{array}$ \\
\hline ALP (U/L) & $\begin{array}{c}73.3 \pm 18.9,70.0 \\
{[36.0-124.0]}\end{array}$ & $\begin{array}{c}69.8 \pm 21.7,64.0 \\
{[29.0-132.0]}\end{array}$ & $\begin{array}{c}63.6 \pm 16.9,62.0 \\
{[26.0-133.0]}\end{array}$ & $\begin{array}{c}60.9 \pm 11.5,62.0 \\
{[26.0-59.0]}\end{array}$ & $\begin{array}{c}62.2 \pm 19.9,59.0 \\
{[27.0-143.0]}\end{array}$ \\
\hline $\mathrm{TB}(\mathrm{mg} / \mathrm{dL})$ & $\begin{array}{c}0.65 \pm 0.39,0.58 \\
{[0.00-1.99]}\end{array}$ & $\begin{array}{c}0.58 \pm 0.33,0.56 \\
{[0.11-1.93]}\end{array}$ & $\begin{array}{c}0.60 \pm 0.31,0.60 \\
{[0.16-1.87]}\end{array}$ & $\begin{array}{c}0.61 \pm 0.32,0.51 \\
{[0.20-1.58]}\end{array}$ & $\begin{array}{c}0.61 \pm 0.29,0.53 \\
{[0.15-1.48]}\end{array}$ \\
\hline $\mathrm{DB}(\mathrm{mg} / \mathrm{dL})$ & $\begin{array}{c}0.15 \pm 0.10,0.14 \\
{[0.00-0.40]}\end{array}$ & $\begin{array}{c}0.17 \pm 0.09,0.15 \\
{[0.04-0.44]}\end{array}$ & $\begin{array}{c}0.20 \pm 0.16,0.19 \\
{[0.03-1.02]}\end{array}$ & $\begin{array}{c}0.18 \pm 0.09,0.16 \\
{[0.03-0.39]}\end{array}$ & $\begin{array}{c}0.18 \pm 0.09,0.17 \\
{[0.04-0.45]}\end{array}$ \\
\hline
\end{tabular}

AST, Aspartate aminotransferase; ALT, Alanine aminotransferase; ALP, Alkaline phosphatase; TB, Total bilirubin; DB, Direct bilirubin.

Table 6. Peak Flow Rate of Subjects before and after Intervention (Mean \pm S.D., $n=39)$

\begin{tabular}{lccc}
\hline \hline \multirow{2}{*}{ Formulation } & \multicolumn{2}{c}{ Peak flow (L/min) } & \\
\cline { 2 - 3 } & Day 1 & Day 7 & \\
\hline Isoniazid & $520 \pm 68$ & $513 \pm 59$ & 0.646 \\
Rifampicin & $539 \pm 49$ & $537 \pm 52$ & 0.215 \\
Pyrazinamide & $566 \pm 74$ & $553 \pm 64$ & 0.690 \\
Levofloxacin & $541 \pm 78$ & $542 \pm 80$ & 0.791 \\
\hline
\end{tabular}

*Paired $t$-test, $\alpha=0.05$.

effects with the study design and the endpoints employed in the observed study. The safety profile of the drugs by inhalation therapy in this study was only $7 d$ and four drugs were given over a period of two months. The oral anti-TB therapy is normally last for 6 months however phase I clinical trial monitors only acute toxicity. The primary safety outcomes (end point safety) i.e., inflammatory cytokines over $7 \mathrm{~d}$ were considered appropriate in this case. Ongoing long term safety and efficacy of the investigational products are being conducted in clinical trial Phase II. The limitation of this study was the viral infection that occurred during the study that could have distorted the results or interpretation especially in the case of the common cold. Also, the number of drugs tested was limited to four common Anti-TB agents.

\section{CONCLUSION}

Administration of anti-TB drugs (isoniazid $5 \mathrm{mg}$, rifampicin $2 \mathrm{mg}$, pyrazinamide $16 \mathrm{mg}$ or levofloxacin $2 \mathrm{mg}$ ) by inhalation using 39 healthy volunteers caused no significant changes in TNF- $\alpha$, IL- $1 \beta$, liver enzymes and kidney functions. The peak flow rate did not change significantly before and after intervention. There were no serious adverse events. The only complaint was recorded by one subject that coughed after first inhalation of the formulation. This probably could have been avoided by better training on how to administer the inhalation. No jaundice or any skin allergies were reported. Although this study used a small sample size, it can be concluded that the inhalation of the four anti-TB dry powders used in this study were safe to use by humans. These inhaled anti-TB drugs have a potential for further investigation of their safety and efficacy in TB patients.
Table 7. Evaluation of Adverse Effects Due to Inhalation of Antituberculosis Formulations

\begin{tabular}{lrrrr}
\hline \hline Reported side effects & Male & Female & Total & Remarks \\
\hline None & 19 & 19 & 38 & \\
Rash & 0 & 0 & 0 & \\
Hepatitis & 0 & 0 & 0 & \\
Nausea/Vomiting & 0 & 0 & 0 & \\
Tingling of extremities & 0 & 0 & 0 & \\
Fatigue & 1 & 0 & 1 & 1 subject had a common \\
Dark urine & & & & cold. \\
Dizziness & 0 & 0 & 0 & \\
Loss of appetite & 0 & 0 & 0 & \\
Ringing in ears & 0 & 0 & 0 & \\
Numbness & 0 & 0 & 0 & \\
Joint pains & 0 & 0 & 0 & \\
Abdominal Pain & 0 & 0 & 0 & \\
Jaundice & 0 & 0 & 0 & \\
Fever & 0 & 0 & 0 & \\
& 1 & 0 & 1 & 1 subject had a common \\
Vision/Hearing change & 0 & 0 & 0 & \\
Behavioral changes & 0 & 0 & 0 & \\
Other & 0 & 0 & 0 & \\
\hline
\end{tabular}

Acknowledgments The authors would like to thank the National Research Council of Thailand (NRCT, Thailand) and Nanotec-PSU center of Excellence on Drug Delivery System for financial support. Instrumentation facility provided by Prince of Songkla University is also thankfully acknowledged. Thanks to Dr. Brian Hodgson for assistance with the English.

Conflict of Interest The authors declare no conflict of interest.

\section{REFERENCES}

1) World Health Organization. Global Tuberculosis Report. WHO Press, France (2015).

2) Misra A, Hickey AJ, Rossi C, Borchard G, Terada H, Makino K, Fourie PB, Colombo P. Inhaled drug therapy for treatment of tuberculosis. Tuberculosis (Edinb.), 91, 71-81 (2011).

3) Labiris NR, Dolovich MB. Pulmonary drug delivery. Part I: physiological factors affecting therapeutic effectiveness of aerosolized 
medications. Br. J. Clin. Pharmacol., 56, 588-599 (2003).

4) Olsson B, Bondesson E, Borgström L, Edsbäcker S, Eirefelt S, Ekelund K, Gustavsson L, Hegelund-Myrbäck T. Pulmonary drug metabolism, clearance, and absorption. Controlled Pulmonary Drug Delivery, 2011, 21-50 (2011).

5) Patton JS, Byron PR. Inhaling medicines: delivering drugs to the body through the lungs. Nat. Rev. Drug Discov., 6, 67-74 (2007).

6) West JB. Respiratory Physiology. The Essentials. Lippincott Williams and Wilkins, Philadelphia (2007).

7) Changsan N, Chan HK, Separovic F, Srichana T. Physicochemical characterization and stability of rifampicin liposome dry powder formulations for inhalation. J. Pharm. Sci., 98, 628-639 (2009).

8) Changsan N, Nilkaeo A, Pungrassami P, Srichana T. Monitoring safety of liposomes containing rifampicin on respiratory cell lines and in vitro efficacy against Mycobacterium bovis in alveolar macrophages. J. Drug Target., 17, 751-762 (2009).

9) Rojanarat W, Changsan N, Tawithong E, Pinsuwan S, Chan HK, Srichana T. Isoniazid proliposome powders for inhalation-preparation, characterization and cell culture studies. Int. J. Mol. Sci., 12, 4414-4434 (2011).

10) Rojanarat $\mathrm{W}$, Nakpheng $\mathrm{T}$, Thawithong E, Yanyium N, Srichana $\mathrm{T}$. Inhaled pyrazinamide proliposome for targeting alveolar macrophages. Drug Deliv., 19, 334-345 (2012).

11) Dharmadhikari AS, Kabadi M, Gerety B, Hickey AJ, Fourie PB, Nardell E. Phase I, single-dose, dose-escalating study of inhaled dry powder capreomycin: a new approach to therapy of drug-resistant tuberculosis. Antimicrob. Agents Chemother., 57, 2613-2619 (2013).

12) Daldegan MB, Teixeira MM, Talvani A. Concentration of CCL11, CXCL8 and TNF-alpha in sputum and plasma of patients undergoing asthma or chronic obstructive pulmonary disease exacerbation. Braz. J. Med. Biol. Res., 38, 1359-1365 (2005).

13) Smountas AA, Lands LC, Mohammed SR, Grey V. Induced sputum in cystic fibrosis: within-week reproducibility of inflammatory markers. Clin. Biochem., 37, 1031-1036 (2004).

14) Obase Y, Shimoda T, Mitsuta K, Matsuo N, Matsuse H, Kohno S. Correlation between airway hyperresponsiveness and airway inflammation in a young adult population: eosinophil, ECP, and cytokine levels in induced sputum. Ann. Allergy Asthma Immunol., 86, 304-310 (2001).

15) Hacievliyagil SS, Gunen H, Mutlu LC, Karabulut AB, Temel I. Association between cytokines in induced sputum and severity of chronic obstructive pulmonary disease. Respir. Med., 100, 846-854 (2006).

16) Hernandez ML, Mills K, Almond M, Todoric K, Aleman MM, Zhang $\mathrm{H}$, Zhou $\mathrm{H}$, Peden DB. IL-1 receptor antagonist reduces endotoxin-induced airway inflammation in healthy volunteers. $\mathrm{J} . \mathrm{Al}$ lergy Clin. Immunol., 135, 379-385 (2015).

17) Li Y, Chongsuvivatwong V, Geater A, Liu A. Exhaled breath condensate cytokine level as a diagnostic tool for obstructive sleep apnea syndrome. Sleep Med., 10, 95-103 (2009).

18) Sack U, Scheibe R, Wotzel M, Hammerschmidt S, Kuhn H,
Emmrich F, Hoheisel G, Wirtz H, Gessner C. Multiplex analysis of cytokines in exhaled breath condensate. Cytometry A, 69A, 169-172 (2006).

19) Rojanarat $W$, Nakpheng $T$, Thawithong E, Yanyium N, Srichana $T$. Levofloxacin-proliposomes: opportunities for use in lung tuberculosis. Pharmaceutics, 4, 385-412 (2012).

20) Bafadhel M, McCormick M, Saha S, McKenna S, Shelley M, Hargadon B, Mistry V, Reid C, Parker D, Dodson P, Jenkins M, Lloyd A, Rugman P, Newbold P, Brightling CE. Profiling of sputum inflammatory mediators in asthma and chronic obstructive pulmonary disease. Respiration, 83, 36-44 (2011).

21) Alving CR. Macrophages as targets for delivery of liposome-encapsulated antimicrobial agents. Adv. Drug Deliv. Rev., 2, 107-128 (1988).

22) Global Alliance for TB Drug Development. Pyrazinamide. Tuberculosis: Handbook of Anti-Tuberculosis Agents, 88, 141-144 (2008).

23) Global Alliance for TB Drug Development. Isoniazid. Tuberculosis: Handbook of Anti-Tuberculosis Agents, 88, 112-116 (2008).

24) British Pharmacopoeia. British Pharmacopoeial Commission, London (2008).

25) Kaewjan K, Srichana T. Nano spray-dried pyrazinamide-l-leucine dry powders, physical properties and feasibility used as dry powder aerosols. Pharm. Dev. Technol., 21, 68-75 (2016).

26) Srichana T, Martin GP, Marriott C. Dry powder inhalers: the influence of device resistance and powder formulation on drug and lactose deposition in vitro. Eur. J. Pharm. Sci., 7, 73-80 (1998).

27) Cook JA, Ramsay CR, Vale LD, DELTA Project Group. Guidance on minimally important clinical difference and trial size is needed. BMJ, 343 (jul25 3), d4375 (2011).

28) Centers for Disease Control and Prevention. Treatment of Tuberculosis, Morbidity and Mortality Weekly Report. American Thoracic Society, CDC, and Infectious Diseases Society of America, Atlanta, GA (2003).

29) Agrawal S, Kaur KJ, Singh I, Bhade SR, Kaul CL, Panchagnula R. Assessment of bioequivalence of rifampicin, isoniazid and pyrazinamide in a four drug fixed dose combination with separate formulations at the same dose levels. Int. J. Pharm., 233, 169-177 (2002).

30) Chideya S, Winston CA, Peloquin CA, Bradford WZ, Hopewell PC, Wells CD, Reingold AL, Kenyon TA, Moeti TL, Tappero JW. Isoniazid, rifampin, ethambutol, and pyrazinamide pharmacokinetics and treatment outcomes among a predominantly HIV-infected cohort of adults with tuberculosis from Botswana. Clin. Infect. Dis., 48, 1685-1694 (2009).

31) Panchagnula R, Sancheti P, Rungta S, Agrawal S, Kaul CL. Evaluation of bioequivalence of isoniazid and pyrazinamide in three and four drugs fixed dose combinations using WHO simplified protocol. Pharmacol. Res., 48, 383-387 (2003).

32) Tappero JW, Bradford WZ, Agerton TB, Hopewell P, Reingold AL, Lockman S, Oyewo A, Talbot EA, Kenyon TA, Moeti TL, Moffat HJ, Peloquin CA. Serum concentrations of antimycobacterial drugs in patients with pulmonary tuberculosis in Botswana. Clin. Infect. Dis., 41, 461-469 (2005). 